\title{
ARTICLE
}

\section{Personalised approaches to pharmacotherapy for schizophrenia}

\author{
John Lally \& James H. MacCabe
}

\begin{abstract}
John Lally is a Clinical Research
Fellow at the Department of

Psychosis Studies, Institute

of Psychiatry, Psychology \&

Neuroscience (loPPN), King's College

London, and National Psychosis

Service, South London and Maudsley

NHS Foundation Trust, London, UK.

James H. MacCabe is Reader in

the Epidemiology of Psychosis at the

Department of Psychosis Studies,

IoPPN, King's College London, and

a consultant psychiatrist, National

Psychosis Service, South London and

Maudsley NHS Foundation Trust,

London, UK.

Correspondence Dr James

H. MacCabe, Reader in the

Epidemiology of Psychosis,

Department of Psychosis Studies,

Institute of Psychiatry, Psychology

$\&$ Neuroscience, King's College

London, De Crespigny Park

London SE5 8AF, UK. Email: james.

maccabe@kcl.ac.uk
\end{abstract}

\begin{abstract}
SUMMARY
The traditional approach to selecting antipsychotic medication involves little more than trial and error. Recent advances in genetics and molecular science offer the hope of a 'personalised medicine' approach to antipsychotic development and prescribing in schizophrenia. Personalised medicine is the practice of tailoring medical treatment to the individual characteristics of each patient. In schizophrenia, this will involve the identification of more homogeneous subsets of patients through the application of genetics, epigenetics, proteomics and metabolomics, neuroimaging and other biomarkers, and the use of these findings to stratify patients according to their response to treatment. In this article, we focus on the emerging evidence in pharmacogenetics and biomarkers for assessing individual response and tolerability of antipsychotic medication in schizophrenia.
\end{abstract}

\section{LEARNING OBJECTIVES}

- Understand the implementation of personalised medicine in general medicine and the implications for psychiatric clinical practice

- Identify benefits and challenges in the implementation of personalised medicine to optimise outcomes to antipsychotic medication

- Evaluate approaches to the use of personalised medicine in the treatment of schizophrenia

\section{DECLARATION OF INTEREST}

None

\section{What is personalised medicine?}

Personalised medicine has been defined as the 'use of genetic or other biomarker information to improve the safety, effectiveness, and health outcomes of patients via more efficiently targeted risk stratification, prevention, and tailored medication and treatment management approaches' (Faulkner 2012).

It is a novel approach to therapeutic decisionmaking, which has particular relevance and potential in schizophrenia, given that individuals with the same diagnosis and receiving the same prescription have high variability in treatment response, leading to the switching of medications multiple times to achieve response.
Biomarkers are defined as characteristics which can be objectively measured and evaluated and used as an indicator of health, disease or pharmacological response to a therapeutic intervention (Biomarkers Definitions Working Group 2001). The ability of a biomarker to predict an individual's response or adverse effects to a particular therapy is central to the implementation of personalised medicine (Weickert 2013). Biomarkers generally refer to genomic or proteomic variations, with neuroimaging, cognitive and psychological variations additionally relevant in schizophrenia. The use of such biomarkers to guide treatment choices can potentially reduce the clinical uncertainty associated with treatment decisions, leading to safer and more effective antipsychotic treatments, as well as increasing the speed of drug development through the focusing of trials on specific patient subsets. At present there are no specific biomarkers to guide the choice of antipsychotic treatment.

\section{Personalised medicine across specialties}

Personalised medicine has been practised in other fields of medicine for many decades (Table 1). For example, arthritis remains a clinical diagnosis, with symptoms and specific clinical signs used to make the diagnosis, but the presence or absence of rheumatoid factor (which is not a diagnostic test) is used to guide the choice of treatment (Kapur 2012). In other diseases, treatment response is used to guide subsequent treatment: for hepatitis $\mathrm{C}$, the response at 12 weeks to interferon-alpha has a positive predictive value of $83 \%$ for predicting viral-free stages at week 24 of treatment and is used to determine which patients will continue with the treatment for a further 12 weeks (Oquendo 2014). Data from a recent prespecified pharmacogenetic testing study indicates that genetic polymorphisms in CYP2C9 and VKORC1 affect the pharmacological and safety outcomes of warfarin therapy. Specifically, CYP2C9 and VKORC1 genotyping could be used in patients to optimise the choice of treatment with warfarin, and to identify sensitive responders who will require a lower dose of warfarin to attain international normalised ratio (INR) levels within the therapeutic range and reduce the likelihood of over-anticogulation and overt bleeding (Mega 2015). 
It is in oncology that personalised medicine has had the most notable successes to date. In the best known example, the identification of the human epidermal growth factor receptor-2 (HER-2), which is overexpressed in approximately $20-30 \%$ of human breast cancers, allows for targeted treatment with the monoclonal antibody trastuzumab. HER2-positive tumours make up around $20 \%$ of breast carcinomas and are generally more aggressive and carry a worse prognosis than HER-2-negative tumours, but the accurate testing for HER-2 positivity allows patients to benefit from the use of trastuzumab, with a reduction in metastatic disease and recurrence (Moja 2012). Trastuzumab is effective for a subset of patients with breast carcinoma with HER-2-positive tumours, but is no better than standard therapy for those with HER-2-negative tumours. This use of a proteomic biomarker profile for the targeting therapeutic interventions was a major landmark in clinical therapeutics for the treatment of breast carcinoma. Such an approach offers hope for psychiatry, indicating that tests predictive of antipsychotic response could be used alongside existing DSM and ICD diagnostic classifications; although the underlying aetiology of the disorder may not be known, treatment stratification can still occur.

\section{Genome-wide association studies (GWAS)}

There has been some success for GWAS outside of psychiatry in validating important genetic variance, with $C Y P 2 C 19^{*} 2$ carriers treated with clopidogrel identified to be at higher risk for cardiovascular adverse events, particularly stent thrombosis, owing to a diminished platelet response to clopidogrel (Sofi 2011). The utility of this finding has been demonstrated in a novel bedside genetic test which has been developed to identify carriers of the $C Y P 2 C 19 * 2$ allele using a buccal swab, helping to detect patients in whom clopidogrel should be avoided (Roberts 2012). This allows for the rapid identification of those who have a risk of being sub-optimally treated, thereby allowing a pharmacogenetic test to be used to guide antiplatelet treatment after percutaneous coronary intervention. This is a demonstration that "point of care' genetic testing can be effectively performed at the bedside, potentially providing an important step towards the use of personalised medicine.

\section{The case for personalised medicine in schizophrenia}

\section{Heterogeneity of clinical response to antipsychotics}

The diagnostic classification of schizophrenia relies on the presence of specific symptoms and the course of the illness, without there being any diagnostically distinguishing molecular or genetic biomarkers to aid the diagnostic process. The phenotypical presentation of schizophrenia is highly heterogeneous, a characteristic that is reflected in the

\section{TABLE 1 Milestones in personalised medicine}

\begin{tabular}{|c|c|c|c|c|}
\hline Study and/or year & Variant & Drug & Effect & Clinical implication \\
\hline Alving et al (1956) & $\begin{array}{l}\text { Glucose-6-phosphate } \\
\text { dehydrogenase (G6PD) } \\
\text { deficiency }\end{array}$ & $\begin{array}{l}\text { Primaquine } \\
\text { (antimalarial drug) }\end{array}$ & $\begin{array}{l}\text { Toxicity due to metabolic enzyme } \\
\text { (G6PD) deficiency }\end{array}$ & $\begin{array}{l}\text { Treatment with primaquine preceded by screening } \\
\text { for G6PD deficiency to prevent toxicity due to } \\
\text { haemolytic anaemia }\end{array}$ \\
\hline Mahgoub et al (1977) & CYP2D6 & $\begin{array}{l}\text { Debrisoquine (anti- } \\
\text { hypertensive) }\end{array}$ & $\begin{array}{l}\text { Poor metabolisers have increased } \\
\text { side-effects }\end{array}$ & $\begin{array}{l}\text { Identified CYP2D6 polymorphism with increased } \\
\text { toxicity secondary to debrisoquine }\end{array}$ \\
\hline Slamon et al(1987) & HER-2 expression & Trastuzumab & $\begin{array}{l}\text { Improved response rate and } \\
\text { progression-free survival in HER- } \\
\text { 2-positive breast cancer }\end{array}$ & $\begin{array}{l}\text { Prediction of response in breast neoplasms/targeted } \\
\text { treatment with a recombinant humanised mono- } \\
\text { clonal antibody for HER-2-postive breast cancer }\end{array}$ \\
\hline \multicolumn{5}{|c|}{ 1998: trastuzumab approved in USA for HER-2-positive breast cancer } \\
\hline \multicolumn{5}{|c|}{ 2003: Human Genome Project completed } \\
\hline Ferrell \& McLeod (2008) & $H L A-B^{*} 1502$ & Carbamazepine & $\begin{array}{l}\text { Increased risk of severe dermato- } \\
\text { logical hypersensitivity reaction }\end{array}$ & $\begin{array}{l}\text { Screening of patients at high risk of } \\
\text { hypersensitivity reactions pre-administration }\end{array}$ \\
\hline Mallal et al(2008) & $H L A-B^{*} 1502$ & Abacavir & $\begin{array}{l}\text { Increased risk of generalised } \\
\text { hypersensitivity reaction }\end{array}$ & $\begin{array}{l}\text { Screening of patients at high risk of } \\
\text { hypersensitivity reactions pre-administration }\end{array}$ \\
\hline Jacobson et al(2011) & Genotype 1 hepatitis C & Telaprevir & & $\begin{array}{l}\text { Telaprevir treatment only for those with genotype } \\
1 \text { hepatitis C }\end{array}$ \\
\hline Finkelman et al(2011) & CYP2C9/VKORC1 & Warfarin & Increased risk of bleeding & $\begin{array}{l}\text { Enhance clinical algorithms to guide warfarin } \\
\text { dosing and lower doses required in sensitive } \\
\text { responders }\end{array}$ \\
\hline $\begin{array}{l}\text { Sofi et al (2011), } \\
\text { Roberts et al (2012) }\end{array}$ & CYP2C19 & Clopidogrel & Increased metabolism & $\begin{array}{l}\text { Point of care genetic testing } \\
\text { Increased risk of post-stent thrombosis after } \\
\text { percutaneous coronary intervention for } \\
\text { CYP2C19*2 carriers }\end{array}$ \\
\hline
\end{tabular}


variability of response to antipsychotic medication in patients with the disorder. Approximately 50$70 \%$ of patients with a first episode of schizophrenia will respond to antipsychotic medication, with this response rate falling to $20 \%$ for those who require a second trial (Agid 2011).

Although antipsychotics are the mainstay of treatment for schizophrenia, about a third of patients do not respond to them and are described as having treatment-resistant schizophrenia. Further, non-adherence to antipsychotic treatment is high and up to $75 \%$ of patients are not adhering to their antipsychotic medication regimen 2 years after discharge from hospital (Leucht 2006). In large part this is due to intolerable adverse effects, leading to the discontinuation of antipsychotics.

\section{The high cost of antipsychotic treatment failure}

Antipsychotic treatment failure and intolerability comes with a high clinical and economic cost. The proportion of patients who recover from schizophrenia is far from impressive, with a recent systematic review estimating that only one in seven patients attain a functional recovery (Jääskeläinen 2013), and only 20-30\% of people with schizophrenia have a complete remission of symptoms (Fenton 1991).

The cost of care for schizophrenia was estimated to be $£ 6.7$ billion in the UK alone in 2004-2005 (Mangalore 2007), and the total societal cost in England is estimated at $£ 11.8$ billion per year, with a cost to the public sector of $£ 7.2$ billion (Andrew 2012). The cost of care for individuals with treatment-refractory schizophrenia is very high, with annual costs for patients with treatmentresistant schizophrenia being 3- to 11-fold higher than for patients with treatment-responsive schizophrenia (which are \$15500-\$22300 per annum in the USA) (Kennedy 2014). These findings reinforce the need to find better ways to predict antipsychotic treatment response and tolerability.

This heterogeneity in treatment response and tolerability in schizophrenia has led to emerging attempts to stratify the patient population by treatment response. In the rest of this review, we will focus on the general application of genomic and other biomarkers for the prediction of treatment response in schizophrenia and on obstacles to personalised medicine for the disorder.

\section{Pharmacogenetics and antipsychotics}

So far there is no evidence that testing for genetic polymorphisms in patients undergoing treatment with antipsychotics leads to improvement in outcomes, but there have been some promising leads with certain pharmacokinetic and pharmacogenetic tests, including the identification of pharmacogenetic changes relating to the cytochrome P450 (CYP450) enzyme system. A systematic review summarised current progress in the pharmacogenetics of antipsychotic medication focusing on studies from the past two decades (Zhang 2011). Most pharmacogenetic studies of antipsychotics have used a candidate gene approach, focusing on polymorphisms in genes coding for receptors in the dopamine and serotonin (5-HT) systems, as well as genes coding for enzymes that metabolise medications, such as catechol-Omethyltransferase (COMT) and CYP450 enzymes (especially CYP2D6), and for transmembrane transporters such as ABCB1. Although numerous studies suggest that antipsychotic efficacy and induced side-effects are associated with candidate gene polymorphisms, most findings are of modest effect, with inconsistent results to date. At present, there is little promising data to indicate that candidate gene testing is applicable to predict antipsychotic efficacy or the risk of antipsychoticinduced side-effects.

Table 2 summarises significant findings from candidate gene studies of antipsychotic efficacy and side-effects.

A recent systematic review identified only a sole $A B C D 1$ gene polymorphism as being predictive of serum clozapine levels and response to clozapine (Krivoy 2015), although further longitudinal studies are required to elucidate the role of $A B C D 1$ in clozapine pharmacokinetics.

The field of GWAS in psychiatry has developed rapidly, but this remains a novel area of research into antipsychotic treatment response. To the best of our knowledge there have only been two studies looking to predict antipsychotic response using a polygenic risk scoring method derived from GWAS (where the risk score is built from combining the cumulative risk of many genetic loci to identify patients most likely to respond). Frank et al (2015) showed that a higher genetic risk for schizophrenia is associated with worse treatment outcomes, mirroring findings from Ikeda et al (2015), who showed that genes related to risperidone response overlap to a modest degree with genes that increase the risk for developing schizophrenia.

The potential benefits and function of pharmacogenetics in schizophrenia are listed in Box 1 and the biomarkers which have shown promise are shown in Box 2.

\section{Pharmacogenetics and treatment-resistant schizophrenia}

The challenges relating to developing pharmacogenetic testing in schizophrenia are outlined in 
TABLE 2 Positive candidate gene studies of treatment response and antipsychotic-induced side-effects in schizophrenia ${ }^{a}$

\begin{tabular}{|c|c|c|c|}
\hline Gene & Variant & Reported association & Medication \\
\hline \multicolumn{4}{|l|}{ Dopamine } \\
\hline DAT & Multiple & & \\
\hline DRD1 & Multiple & & \\
\hline \multirow[t]{2}{*}{ DRD2 } & Taq B & Improved psychotic symptoms & Clozapine \\
\hline & Ser311Cys & Ser 311 homozygotes with poorer response & Risperidone \\
\hline \multirow[t]{2}{*}{ DRD3 } & $-241 \mathrm{~A} / \mathrm{G}$ & Increased efficacy in those with $-241 \mathrm{~A}$ allele & Risperidone \\
\hline & SergGly & $\begin{array}{l}\text { Ser/Ser allele associated with poorer response } \\
\text { Increased efficacy in those with Ser allele }\end{array}$ & $\begin{array}{l}\text { SGAs } \\
\text { Clozapine, risperidone }\end{array}$ \\
\hline \multicolumn{4}{|l|}{ Serotonin } \\
\hline \multirow[t]{2}{*}{$5-\mathrm{HT} 2 \mathrm{~A}$} & $102 \mathrm{~T} / \mathrm{C}$ & $\begin{array}{l}\text { C/C genotype: with improved response } \\
\text { Poorer response }\end{array}$ & $\begin{array}{l}\text { Risperidone } \\
\text { Clozapine }\end{array}$ \\
\hline & $-1438 \mathrm{G} / \mathrm{A}$ & $\begin{array}{l}\mathrm{G} / \mathrm{G} \text { genotype poorer response } \\
\mathrm{G} \text { allele improved response }\end{array}$ & $\begin{array}{l}\text { Clozapine and FGAs } \\
\text { FGAs and SGAs }\end{array}$ \\
\hline \multirow[t]{2}{*}{$5-\mathrm{HT} 2 \mathrm{C}$} & His 453Tyr & Tyr associated with non-response & Clozapine \\
\hline & Cys23Ser & Ser associated with increased efficacy & Clozapine \\
\hline \multirow[t]{2}{*}{ 5-HT6 } & VNTR & Short allele associated with improved response & Clozapine \\
\hline & $267 \mathrm{~T} / \mathrm{C}$ & Improved response in $\mathrm{T} / \mathrm{T}$ genotype & Clozapine, risperidone \\
\hline \multicolumn{4}{|l|}{ Antipsychotic-induced side-effects } \\
\hline \multicolumn{4}{|l|}{ Metabolic disturbances } \\
\hline \multirow[t]{2}{*}{$5-\mathrm{HT} 2 \mathrm{C}$} & $759 \mathrm{C} / \mathrm{T}$ & $\begin{array}{l}\mathrm{C} \text { allele associated with weight gain and } \mathrm{T} \text { allele with } \\
\text { a protective effect }\end{array}$ & Clozapine, olanzapine \\
\hline & $\begin{array}{l}\text { Cys23Ser- polymorphisms } \\
\text { rs518147, rs1414334, and } \\
5 \text { - } \mathrm{HTR}_{2 \mathrm{C}} \mathrm{C} . \mathrm{C} .1-142948(\mathrm{GT}) \mathrm{n}\end{array}$ & Increased risk of metabolic syndrome & $\begin{array}{l}\text { Clozapine, olanzapine, } \\
\text { risperidone }\end{array}$ \\
\hline Leptin gene & $-2548 \mathrm{~A} / \mathrm{G}$ (GG/GA genotype) & Increased weight gain and risk of metabolic syndrome & Clozapine, olanzapine \\
\hline Melanocortin 4 receptor gene (MC4R) & $\begin{array}{l}\text { rs17782313 C allele (CC genotype) } \\
\text { rs8087522 A allele }\end{array}$ & Increased weight gain & Clozapine \\
\hline DRD2 & rs4436578 C allele & Increased weight gain & Clozapine \\
\hline
\end{tabular}

FGA, first-generation antipsychotic; SGA, second-generation antipsychotic.

a. Although numerous studies suggest that antipsychotic-induced side-effects and antipsychotic efficacy are associated with candidate gene polymorphisms, most findings are of modest effect, with inconsistent results to date (i.e. multiple negative studies of candidate genes exist). This table contains some of the candidate gene studies which have been associated with antipsychotic response or antipsychotic-induced side-effects. Adapted from Arranz et al (2011) and Raja \& Raja (2014).

Box 3. Some of these challenges are diminished when treatment-resistant schizophrenia is considered. Characteristics of treatment-resistant schizophrenia such as increased uniformity in diagnosis, the ability to monitor adherence from serum clozapine levels, the long-term follow-up of patients

\section{BOX 1 Uses of pharmacogenetic biomarkers} in schizophrenia

Pharmacogenomic biomarkers could provide tools to:

- avoid toxicity and subsequent adverse drug reactions

- avoid underdosing and subsequent lack of efficacy

- avoid giving a drug to hypersensitive individuals

- improve clinical diagnosis

- rescue drugs previously withdrawn because of adverse drug reactions by clinical services and the existence of registers for clozapine monitoring services, which make individuals with treatment-resistant schizophrenia a potentially more suitable population in which to conduct pharmacogenetic studies.

\section{BOX 2 Biomarkers of antipsychotic response or risk of antipsychotic toxicity}

The biomarkers that have shown promise in predicting antipsychotic response or toxicity are mostly genetic variants of:

- drug-metabolising enzymes such as CYP2D6, CYP1A2, CYP3A4, CYP2C9, CYP2D6, and COMT

- $\mathrm{ABCD} 1$

- drug targets such as dopaminergic and serotonergic receptors

- human leukocyte antigens (HLA alleles) 
Furthermore, a particular urgency exists in that there are no evidence-based effective treatments for the $30 \%$ of treatment-resistant patients who fail to respond to clozapine (Meltzer 1992; Chakos 2001) or in those in whom clozapine is discontinued because of adverse events. Last, the ability to predict in advance whether or not a given patient is likely to respond to clozapine has the potential to substantially reduce the delay in clozapine initiation.

\section{Pharmacogenetic testing and clozapine response}

Pharmacogenetic studies have investigated the importance of serotonergic and dopaminergic targeting for the antipsychotic effect of clozapine, but the results are conflicting and poorly replicated (Zhang 2011). No single polymorphism can be used for the prediction of clozapine response. The failed attempts to implement findings of candidate gene studies in predicting clozapine response is emblematic of the difficulties in the clinical utilisation of this testing in psychiatry. The lack of translational impact of candidate gene testing in predicting the efficacy of clozapine and other antipsychotics has been a hallmark of studies in this area and has led to more recent efforts to utilise GWAS and polygenic risk scores to provide more robust genetic markers.

\section{Other putative biomarkers}

\section{Neuroimaging findings as biomarkers}

Magnetic resonance spectroscopy has demonstrated that those who show a poor response to antipsychotic treatment in first-episode psychosis have higher levels of glutamate in the anterior cingulate cortex than patients who respond well. Further, high glutamate levels are associated with increased levels of negative symptoms and poor level of functioning (Egerton 2012). These findings were replicated in treatment-resistant patients (Demjaha 2014), parallel to findings using positron emission tomography (PET) in the same individuals, which indicated that dopamine function is relatively normal (Demjaha 2012). These observations suggest that the persistence of symptoms in treatment-resistant patients may be associated with elevated anterior cingulate glutamate levels.

There is some evidence that perfusion imaging may be predictive of response to clozapine; in previous single photon emission computerised tomography (SPECT) studies, symptomatic improvement following clozapine use has been associated with higher levels of resting state perfusion in the striatum, thalamus and prefrontal cortex prior to treatment (Rodriguez 1997). There
BOX 3 Challenges in using pharmacogenetic testing to predict antipsychotic effects in schizophrenia

Characterising/quantifying drug response

- Measuring antipsychotic efficacy

- Non-adherence

- Treatment duration: early benefits with antipsychotic response $v$. longer duration of effect with clozapine

- Concurrent medication use: mood stabilisers; cross-over of antipsychotics during drug switching

Patient characteristics

- Differential diagnosis and unclear diagnostic boundaries

- Illness course: first-episode psychosis and treatmentresistant schizophrenia

- Comorbidities: substance misuse, depression

- Risk of neutropenia increased in African-Caribbeans

- Baseline characteristics: low body mass index in firstepisode psychosis and greater risk of weight gain with antipsychotic treatment

have been previous PET and SPECT studies which have shown frontal and perfusion decreases during clozapine treatment (Molina 2005) and that this decrease is greater in responders than non-responders (Rodriguez 1997). Subsequent discriminate analysis correctly identified $79 \%$ of cases $(80 \%$ of clozapine non-responders and $78 \%$ of clozapine responders) according to thalamic and right prefrontal/cerebellum perfusion ratios prior to starting clozapine (Rodriguez 1997), although this classification rate is not high enough to be clinically useful.

These findings, although remaining preliminary, suggest that treatment outcome in patients with schizophrenia may be predicted by neuroimaging. However, the actual clinical utility of such tests remains to be shown.

Homovanillic acid as a proteomic biomarker

Higher plasma levels of homovanillic acid, a major circulating dopamine metabolite, are generally associated with good subsequent response to antipsychotic treatment, whereas lower dopamine metabolite levels are associated with poor response (Pickar 1986; Davila 1988). A time-dependent decrease in homovanillic acid is correlated with antipsychotic response in most studies, a change which is not seen in patients with treatmentresistant schizophrenia. This is one of the best replicated proteomic biomarkers of antipsychotic treatment response in schizophrenia, although 
discrepant findings exist (Stone 2010). The use of homovanillic acid as a biomarker is limited by the use of plasma homovanillic acid measures in many studies, which is at best an indirect measure of brain activity, given that it is influenced by peripheral as well as central dopamine metabolism.

\section{Pharmacogenetics and adverse reactions}

\section{Medication hypersensitivity}

Although there are few examples of the use of pharmacogenetic testing to maximise treatment response in clinical practice, pharmacogenetics is more widely used in the prediction of hypersensitivity to certain medications such as carbamazepine. In people of South East Asian ethnicity, positivity for the $H L A-B^{*} 1502$ allele conferred an odds ratio of 2504 for developing carbamazepine-induced Stevens-Johnson syndrome/toxic epidermal necrolysis (Ferrell 2008). Similarly, screening for $H L A-B^{\star} 5701$ reduces the risk of a hypersensitivity reaction to abacavir in HIV treatment, with positivity for the allele providing $100 \%$ specificity for the development of an hypersensitivity reaction (Mallal 2008). Both are examples of how a pharmacogenetic test can be used to avert a specific medication toxic effect.

\section{Antipsychotic adverse effects}

The prediction and avoidance of adverse events is an important goal in the use of antipsychotic medication. Two of the most widely studied adverse effects in pharmacogenetics are tardive dyskinesia and weight gain.

Probably the most robust evidence for a genetic finding associated with side-effects is that for antipsychotic-induced weight gain, with the 759T/C polymorphism in the promoter region of the HTR2C gene (rs3813929) associated with a greater than twofold increased risk of weight gain (Arranz 2011). This effect is especially marked with olanzapine and clozapine, both of which have strong affinity to the 5-HT2C receptor.

With regard to tardive dyskinesia, despite several studies of dopamine receptor polymorphisms, the results have been inconsistent and there remains no pharmacogenetic test for the development of antipsychotic-induced tardive dyskinesia (Ozomaro 2013).

\section{Clozapine and agranulocytosis}

Clozapine is underprescribed and its use is limited by patients' and clinicians' fears about the emergence of life-threatening adverse events, in particular agranulocytosis. The mechanism of this idiosyncratic drug reaction is yet to be elucidated, although it is certainly multifactorial, with some evidence that genetic variance increases individuals' susceptibility to clozapine-induced agranulocytosis. It remains an area which has not been extensively studied and, owing to the relatively low incidence of clozapine-induced agranulocytosis, it is dominated by small-sample studies.

The most replicated genetic evidence is for variation in the human leucocyte antigen (HLA) system. This includes several genetic variants involved in the $H L A-D Q B 1$ locus, including associations at two HLA amino acids (a single amino acid at $H L A-D Q B 1(126 Q)$ and an amino acid change in the extracellular binding pocket of HLAB(158T)) (Goldstein 2014). Previously, a casecontrol cohort found that the odds of developing clozapine-induced agranulocytosis were 16.9 times higher in patients with the sequence variant $6672 \mathrm{G}>\mathrm{C}$ on the HLA-DQB1 gene (Athanasiou 2011). This single nucleotide polymorphism has been incorporated into commercially available tests, with test sensitivity and specificity calculated to be $21.5 \%$ and $98.4 \%$ respectively, indicating that about one in five patients at risk of clozapineinduced agranulocytosis would be identified by the test (Athanasiou 2011). Although the HLA-DQB1 locus may be implicated in the development of clozapine-induced agranulocytosis, for the test to have clinical utility for this adverse event, which has a prevalence of $0.8 \%$, it requires high sensitivity as well as specificity. The low sensitivity of the test has been a limiting factor in its application in clinical settings; and it has not been a commercial success (Chowdhury 2011; Verbelen 2015).

\section{Obstacles to personalised medicine and pharmacogenetic testing in schizophrenia}

\section{Complexity and heterogeneity of the schizophrenia phenotype}

Thus far, there has been little success in developing personalised medicine for schizophrenia. The reasons for this are manifold; not least that schizophrenia is a highly complex disorder influenced by multiple combinations of genes and molecular variances acting in the midst of even less clearly definable social and environmental factors, thus limiting the likelihood that a single biological risk factor will map onto a diagnosis of a psychotic disorder.

\section{Lack of a mechanistic understanding of antipsychotics}

The medication response phenotypes in psychiatry are based on clinical observation and not on the physiological processes or biomarkers that can be utilised in other medical specialties such as oncology and cardiovascular medicine. Our limited 
understanding of the mechanism of action of antipsychotics (compared with chemotherapeutic agents in oncology, for example), limits our ability to generate mechanistic hypotheses as to which biomarkers might be relevant to treatment response.

\section{Clinical guidelines and interpretation}

It is noteworthy that there are 121 known pharmacogenetic markers for medications currently listed for prescription by the US Food and Drug Administration, with a sizable minority $(n=26)$ for psychiatric medications (Hamilton 2015). However, specific and clear guidelines for the clinical use of these pharmacogenetic markers are lacking. This highlights a recurring problem, not only in psychiatry, but across other medical specialties as well.

The most common psychotropic medication markers relate to CYP2D6 enzymes. This particular focus for pharmacogenetic research on CYP2D6 genotypes is not unexpected, given that this enzyme metabolises a number of antipsychotics, with most of the research focused on the relationship with risperidone use. Genotyping for CYP enzyme deficiencies could potentially offer a relatively simple solution for optimising antipsychotic medication dosing, although this has not been consistently borne out in practice and genetic testing for CYP polymorphisms is not currently in routine use for guiding antipsychotic choice or dosage. The predictive value of currently available tests is not sufficient to allow the integration of pharmacogenetic testing into clinical guidelines.

\section{Clinician and public attitudes}

Although clinicians and patients are generally positively disposed to the concept of personalised medicine, the introduction of personalised approaches will require a profound change in prescribing practice, with treatment decisions taken out of the hands of clinicians and patients and devolved to algorithms based on genetic tests or other biomarkers. This represents a growing challenge for medical education, if future generations of doctors are to be prepared for advances in the field (Slaughter 2012).

\section{Communication and interpretation}

The communication of pharmacogenetic test results is a critical step towards their widespread utilisation and this has been highlighted across medical specialties. In the future, the focus will shift from the costs of genotyping to the interpretation and production of reports for clinical use. The need to organise and interpret complex pharmacogenetic testing will rely on the utilisation of more highly integrated electronic patient records, to ensure that this information is available for all clinicians during any future clinical encounter.

Positive pharmacogenetic test results will have lifelong implications for a patient's response to, or tolerance of, antipsychotic medication. However, in a fragmented healthcare system, there is a risk that access to pharmacogenetic test information will be limited and that it will be lost to clinicians as the patient moves from one healthcare setting to another.

\section{'Orphan diseases'}

A wider ethical question surrounds the potential for the creation of so-called 'orphan diseases' or, in patients with schizophrenia, a subset of patients with 'Cinderella psychoses'. The implementation of reliable pharmacogenetic testing will inevitably result in the subdivision of patients into smaller subgroups. This would bring with it the possibility that some of these subgroups might be considered less profitable by the pharmaceutical industry, and thus stand a reduced chance of having treatments developed for them, particularly where markers are found that define patients with a low probability of treatment response. Further, there may be potential difficulties in finding adequate numbers of specific patient groups in order to identify variance in individual biomarker profiles. An example of this is the identification of patients at risk for clozapineinduced agranulocytosis, which due to its low incidence and unpredictable occurrence makes it difficult to assemble statistically adequate samples for prospective study. Patients with less profitable genotypes are therefore at risk of becoming therapeutic orphans.

\section{Health economics}

The cost-effectiveness of any personalised medication approach will depend on the prevalence of test-positive individuals, the accuracy and the cost of conducting the test, the predictive validity of the test and the cost of any newly developed medication as a result of the test (Flowers 2004).

There is little research assessing the economic evaluation of the costs and outcomes of the use of pharmacogenetic tests in schizophrenia. We located one recent evaluation of the potential use of a pharmacogenetic test assessing CYP450 polymorphisms prior to treatment with risperidone (Rejon-Parrilla2014).In this study,pharmacogenetic testing prior to risperidone treatment was used to guide dosing and was compared with the usual 'trial and error' dosing scheme. The patient stratification strategy by pharmacokinetic testing improved health compared with traditional dosing schemes at 
a cost of $£ 2059$ per patient. This study's results are promising and indicate that the pharmacogenetic test for risperidone in schizophrenia could meet the requirements of cost-effectiveness set by the National Institute for Health and Care Excellence (NICE). One counter-argument to this approach is that it may be more cost-effective simply to treat with antipsychotics for which common CYP450 variation has little or no effect (e.g. amisulpride).

The stratification of patients with psychotic illness into more refined subsets may lead to increased efficiency of medication development, but with a reduction in the size of the total schizophrenia population. This may lead to the development of multiple subsets of patients and niche products, which will risk segmenting the market and increasing drug development costs (Evers 2009), resulting in more expensive medications for small patient groups, which will not be economically viable products. This may in turn act as a barrier to future medication development by pharmaceutical companies.

\section{Conclusions}

We have described the potential value of personalised medicine in schizophrenia, identified a number of conceptual and practical obstacles to the development of personalised medicine in schizophrenia therapeutics, and have reviewed some of the successful implementations of stratified medicine in other fields, as well as the limited success to date in implementing such approaches in schizophrenia. We believe that with the rapid advance of genetic and other biological tests, there is a compelling case to pursue this field of research for the development of treatments and optimising the use of currently available antipsychotic medication. The integration of personalised medicine into schizophrenia treatment will involve multiple research strands, including neuroimaging and molecular and genetic methods, to improve treatment response and identify toxicity phenotypes in schizophrenia.

\section{Funding}

J.H.M. is supported by STRATA (MRC grant no. MR/L011794) and by the National Institute for Health Research (NIHR) Biomedical Research Centre for Mental Health at the South London and Maudsley NHS Foundation Trust and Institute of Psychiatry, Psychology and Neuroscience, Kings College London.

\section{References}

Agid 0, Arenovich T, Sajeev G, et al (2011) An algorithm-based approach to first-episode schizophrenia: response rates over 3 prospective antipsychotic trials with a retrospective data analysis. Journal of Clinical Psychiatry, 72: 1439-44.

Alving AS, Carson PE, Flanagan CL, et al (1956) Enzymatic deficiency in primaquine-sensitive erythrocytes. Science, 124: 484-5.

Andrew AKM, McCrone P, Parsonage M, et al (2012) Effective Interventions in Schizophrenia: The Economic Case. London School of Economics and Political Science.

Arranz MJ, Rivera M, Munro JC (2011) Pharmacogenetics of response to antipsychotics in patients with schizophrenia. CNS Drugs, 25 : 933-69.

Athanasiou MC, Dettling M, Cascorbi I, et al (2011) Candidate gene analysis identifies a polymorphism in HLA-DOB1 associated with clozapine-induced agranulocytosis. Journal of Clinical Psychiatry, 72 : 458-63.

Biomarkers Definitions Working Group (2001) Biomarkers and surrogate endpoints: preferred definitions and conceptual framework. Clinical Pharmacology and Therapeutics, 69: 89-95.

Chakos M, Lieberman J, Hoffman E, et al (2001) Effectiveness of second-generation antipsychotics in patients with treatment-resistant schizophrenia: a review and meta-analysis of randomized trials. American Journal of Psychiatry, 158: 518-26.

Chowdhury NI, Remington G, Kennedy JL (2011) Genetics of antipsychoticinduced side effects and agranulocytosis. Current Psychiatry Reports, 13: 156-65.

Davila R, Manero E, Zumarraga M, et al (1988) Plasma homovanillic acid as a predictor of response to neuroleptics. Archives of General Psychiatry, 45: $564-7$

Demjaha A, Murray RM, McGuire PK, et al (2012) Dopamine synthesis capacity in patients with treatment-resistant schizophrenia. American Journal of Psychiatry, 169: 1203-10.

Demjaha A, Egerton A, Murray RM, et al (2014) Antipsychotic treatment resistance in schizophrenia associated with elevated glutamate levels but normal dopamine function. Biological Psychiatry, 75: e11-3.

Egerton A, Brugger S, Raffin M, et al (2012) Anterior cingulate glutamate levels related to clinical status following treatment in first-episode schizophrenia. Neuropsychopharmacology, 37: 2515-21.

Evers K (2009) Personalized medicine in psychiatry: ethical challenges and opportunities. Dialogues in Clinical Neuroscience, 11: 427-34

Faulkner E, Annemans L, Garrison L, et al (2012) Challenges in the development and reimbursement of personalized medicine-payer and manufacturer perspectives and implications for health economics and outcomes research: a report of the ISPOR personalized medicine special interest group. Value Health, 15: 1162-71.

Fenton WS, McGlashan TH (1991) Natural history of schizophrenia subtypes. II. Positive and negative symptoms and long-term course. Archives of General Psychiatry, 48: 978-86.

Ferrell Jr PB, McLeod HL (2008) Carbamazepine, HLA-B*1502 and risk of Stevens-Johnson syndrome and toxic epidermal necrolysis: US FDA recommendations. Pharmacogenomics, 9: 1543-6.

Finkelman BS, Gage BF, Johnson JA, et al (2011) Genetic warfarin dosing: tables versus algorithms. Journal of the American College of Cardiology, 57: $612-8$

Flowers CR, Veenstra D (2004) The role of cost-effectiveness analysis in the era of pharmacogenomics. Pharmacoeconomics, 22: 481-93.

Frank J, Lang M, Witt SH, et al (2015) Identification of increased genetic risk scores for schizophrenia in treatment-resistant patients. Molecular Psychiatry, 20: 150-1.

Goldstein Jl, Jarskog LF, Hilliard C, et al (2014) Clozapine-induced agranulocytosis is associated with rare HLA-DQB1 and HLA-B alleles. Nature Communications, 5: 4757.

Hamilton SP (2015) The promise of psychiatric pharmacogenomics. Biological Psychiatry, 77: 29-35

Ikeda M, Yoshimura R, Hashimoto R, et al (2015) Genetic overlap between antipsychotic response and susceptibility to schizophrenia. Journal of Clinical Psychopharmacology, 35: 85-8.
MCO answers

1 b $\quad 2$ e $\quad 3$ a $\quad 4 d \quad 5 c$ 
Jääskeläinen E, Juola P, Hirvonen N, et al (2013) A systematic review and meta-analysis of recovery in schizophrenia. Schizophrenia Bulletin, 39: 1296-306.

Jacobson IM, McHutchison JG, Dusheiko G, et al (2011) Telaprevir for previously untreated chronic hepatitis $C$ virus infection. New England Journal of Medicine, 364: 2405-16.

Kapur S, Phillips AG, Insel TR (2012) Why has it taken so long for biological psychiatry to develop clinical tests and what to do about it? Molecular Psychiatry, 17: 1174-9.

Kennedy JL, Altar CA, Taylor DL, et al (2014) The social and economic burden of treatment-resistant schizophrenia: a systematic literature review. International Clinical Psychopharmacology, 29: 63-76.

Krivoy A, Gaughran F, Weizman A, et al (2015) Gene polymorphisms potentially related to the pharmacokinetics of clozapine: a systematic review. International Clinical Psychopharmacology, 2: 2

Leucht S, Heres S (2006) Epidemiology, clinical consequences, and psychosocial treatment of nonadherence in schizophrenia. Journal of Clinical Psychiatry, 5: 3-8.

Mahgoub A, Idle JR, Dring LG, et al (1977) Polymorphic hydroxylation of debrisoquine in man. Lancet, 2: 584-6.

Mallal S, Phillips E, Carosi G, et al (2008) HLA-B*5701 screening for hypersensitivity to abacavir. New England Journal of Medicine, 358 : 568-79

Mangalore R, Knapp M (2007) Cost of schizophrenia in England. Journal of Mental Health Policy and Economics, 10: 23-41.

Mega JL, Walker JR, Ruff CT, et al (2015) Genetics and the clinical response to warfarin and edoxaban: findings from the randomised, double-blind ENGAGE AF-TIMI 48 trial. Lancet, 385: 2280-7.

Meltzer HY (1992) Treatment of the neuroleptic-nonresponsive schizophrenic patient. Schizophrenia Bulletin, 18: 515-42.

Moja L, Tagliabue L, Balduzzi S, et al (2012) Trastuzumab containing regimens for early breast cancer. Cochrane Database of Systematic Reviews, 4: CD006243.

Molina V, Gispert JD, Reig S, et al (2005) Cerebral metabolic changes induced by clozapine in schizophrenia and related to clinical improvement. Psychopharmacology (Berlin), 178: 17-26.

Oquendo MA, McGrath P, Weissman MM (2014) Biomarker studies and the future of personalized treatment for depression. Depression and Anxiety, 31: 902-5.
Ozomaro U, Wahlestedt C, Nemeroff CB (2013) Personalized medicine in psychiatry: problems and promises. BMC Medicine, 11: 132.

Pickar D, Labarca R, Doran AR, et al (1986) Longitudinal measurement of plasma homovanillic acid levels in schizophrenic patients: correlation with psychosis and response to neuroleptic treatment. Archives of General Psychiatry, 43: 669-76.

Raja M, Raja S (2014) Clozapine safety, 40 years later. Current Drug Safety, 9: 163-95.

Rejon-Parrilla JC, Nuijten M, Redekop WK, et al (2014) Economic evaluation of the use of a pharmacogenetic diagnostic test in schizophrenia. Health Policy and Technology, 3: 314-24.

Roberts JD, Wells GA, Le May MR, et al (2012) Point-of-care genetic testing for personalisation of antiplatelet treatment (RAPID GENE): a prospective, randomised, proof-of-concept trial. Lancet, 379: 1705-11.

Rodriguez VM, Andree RM, Castejon MJ, et al (1997) Fronto-striatothalamic perfusion and clozapine response in treatment-refractory schizophrenic patients. A 99mTc-HMPAO study. Psychiatry Research, 76: 51-61.

Slamon DJ, Clark GM, Wong SG, et al (1987) Human breast cancer: correlation of relapse and survival with amplification of the HER-2/neu oncogene. Science, 235: 177-82.

Slaughter RL (2012) Translation of pharmacogenetics to clinical practice: what will it take? Expert Review of Clinical Pharmacology, 5: 101-3.

Sofi F, Giusti B, Marcucci R, et al (2011) Cytochrome P450 $2 \mathrm{C} 19^{*} 2$ polymorphism and cardiovascular recurrences in patients taking clopidogrel: a meta-analysis. Pharmacogenomics Journal, 11: 199-206.

Stone JM, Raffin M, Morrison P, et al (2010) Review: the biological basis of antipsychotic response in schizophrenia. Journal of Psychopharmacology, 24: 953-64.

Verbelen M, Collier DA, Cohen D, et al (2015) Establishing the characteristics of an effective pharmacogenetic test for clozapine-induced agranulocytosis. Pharmacogenomics Journal, 15: 461-6.

Weickert CS, Weickert TW Pillai A, et al (2013) Biomarkers in schizophrenia: a brief conceptual consideration. Disease Markers, 35: 7.

Zhang JP, Malhotra AK (2011) Pharmacogenetics and antipsychotics: therapeutic efficacy and side effects prediction. Expert Opinion on Drug Metabolism and Toxicology, 7: 9-37.

\section{MCOs}

Select the single best option for each question stem

1 All of the following are true of biomarkers in schizophrenia except:

a specific biomarkers can be used to aid diagnosis

b specific biomarkers exist to guide choice of antipsychotic medication

c specific biomarkers are used in clinical practice for disease prognosis

d specific biomarkers are used in clinical practice to determine treatment response

e specific biomarkers are used in clinical practice to enhance safety of medication

2 Clozapine response has been shown to be predicted by examining genetic polymorphisms in:

a $\mathrm{H}_{2}$ receptors

b $5-\mathrm{HT}_{2 \mathrm{~A}}$ receptors c $5-\mathrm{HT}_{2 \mathrm{C}}$ receptors

d alpha-2 receptors

e none of the above.

3 Cytochrome enzyme polymorphisms can affect all of the following except:

a drug absorption

b drug efficiency

c drug interactions

d drug serum levels

e drug-related adverse events.

4 Potential outcomes of the successful implementation of pharmacogenetic testing include all of the following except:

a development of new antipsychotic medications

b lower rates of adverse events with antipsychotics

c stratification of patients into subgroups, some of which could be neglected in research d lower rates of clinical response to antipsychotics

e tailored utilisation of extant antipsychotics

5 Biomarkers and pharmacogenetic testing are used clinically with the following medications except:

a clopidogrel

b carbamazepine

c olanzapine

d abacavir

e trastuzumab. 